\title{
A Novel Calibration Method Research of the Scale Factor for the All-optical Atomic Spin Inertial Measurement Device
}

\author{
Sheng Zou ${ }^{1}$, Hong Zhang ${ }^{1}$, Xi-yuan Chen ${ }^{1}$, Yao Chen ${ }^{2}$, and Jian-cheng Fang ${ }^{2}$ \\ ${ }^{1}$ Key Laboratory of Micro-Inertial Instrument and Advanced Navigation Technology, Ministry of \\ Education, School of Instrument Science and Engineering, Southeast University, Sipailou 2, \\ Nanjing 210096, China \\ ${ }^{2}$ School of Instrument Science and Opto-Electronics Engineering, Beihang University, Beijing 100191, China
}

(Received March 23, 2015 : revised June 9, 2015 : accepted July 6, 2015)

\begin{abstract}
A novel method to measure the scale factor for the all-optical atomic spin inertial measurement device (ASIMD) is demonstrated in this paper. The method can realize the calibration of the scale factor by a self-consistent method with small errors in the quiescent state. At first, the matured IMU (inertial measurement unit) device was fixed on an optical platform together with the ASIMD, and it has been used to calibrate the scale factor for the ASIMD. The results show that there were some errors causing the inaccuracy of the experiment. By the comparative analysis of theory and experiment, the ASIMD was unable to keep pace with the IMU. Considering the characteristics of the ASIMD, the mismatch between the driven frequency of the optical platform and the bandwidth of the ASIMD was the major reason. An all-optical atomic spin magnetometer was set up at first. The sensitivity of the magnetometer is ultra-high, and it can be used to detect the magnetization of spin-polarized noble gas. The gyromagnetic ratio of the noble gas is a physical constant, and it has already been measured accurately. So a novel calibration method for scale factor based on the gyromagnetic ratio has been presented. The relevant theoretical analysis and experiments have been implemented. The results showed that the scale factor of the device was $7.272 \mathrm{~V} / \%$ s by multi-group experiments with the maximum error value $0.49 \%$.
\end{abstract}

Keywords: Scale factor, All-optical, Atomic spin, Inertial measurement device

OCIS codes : (230.1150) All-optical devices; (230.5160) Photo detectors; (280.4788) Optical sensing and sensors; (020.1335) Atom optics

\section{INTRODUCTION}

With the development of optical pumping technology [1, 2], spin-polarized atoms have been used in many practical applications, such as bio-magnetism atomic sensors [3], atomic magnetometers [4], atomic gyroscopes [5], and so on. The gyroscope is one of the necessary sensors for the Inertial Navigation System (INS), which plays an important role in many facilities which need accurate position, velocity, and orientation information. The inertial measurement device based on spin-polarized atoms has become a hot spot. Spinpolarized atoms will precess at an atomic-species-dependent frequency when subjected to an external magnetic field [6]. The physical rotation of the device containing such atoms will modify the frequency in proportion to the rotation rate. The novel device makes use of detecting the precession frequency to measure the rotation rate [7]. According to the theoretical analysis and experimental results, the ASIMD has many advantages [8-11]. Moreover, the nature of the device's structure is inherently immune to external vibration. Because the ASIMD replaces the mechanical rotor with spin-polarized atoms, it does not have any mechanical collisions and friction, and it offers promise for applications with high precision [5]. The angle-random walk (ARW) of $0.002 \% \sqrt{ } \mathrm{Hz}$ and the bias instability of $0.04 \% \mathrm{~h}$ based on the atomic spin gyroscope have been achieved and it has the potential advantage to achieve the best performance gyroscope in the future [12].

\footnotetext{
*Corresponding author: chxiyuan@seu.edu.cn

Color versions of one or more of the figures in this paper are available online.
} 
The output signal of the IMU has a definite proportional relationship with the attitude angular velocity of the moving carrier. The degree of the IMU's accuracy affects the precision of the INS directly. Due to the influence of many factors such as machining processes, the external environment and so on, the characteristics of the relationship between the gyroscope's input and output signals is nonlinear. It leads to errors in the scale factor. At present, many scholars have made contributions in the calibration of the scale factor. Some researchers established the relevant mathematic model of scale factor on full range and put forward the corresponding compensated methods [13, 14]. Others carried out their research aiming at the characteristics of scale factor error such as asymmetric positions, nonlinear problems and so on $[15,16]$. Some research works have been proved valid by the experimentally verified results, and the errors have been reduced in different degrees [13-16]. The method of using the high precision IMU as a standard has been generally adopted in these traditional methods to determine the scale factor, and most of the research aimed at the common gyroscopes and focused on the mathematical process without the considerations of the work principle of the device.

The ASIMD is a new kind of gyroscope, and it is still in the stage of development without official methods for scale factor calibration. In this paper, we have calibrated the scale factor for the device based on the mature product (IMU) at first. However, the experimental results have been influenced by many factors such as the precision control of the frequency for the optical platform, the precision adjustment of the amplitude for the platform, the decrease of system errors and so on. In the view of these problems, we have changed the test method. According to the characteristics of the device, we calibrated the scale factor based on the gyromagnetic ratio of the noble gas, and it has been tested accurately by the related theoretical analysis and experimental verification.

\section{PRINCIPLE OF ALL-OPTICAL ATOMIC SPIN INERTIAL MEASUREMENT DEVICE}

The basic theory of the ASIMD is based on the effect of nuclear magnetic resonance $[10,12]$. The principle can be described as follows:

$$
\begin{aligned}
& \frac{\partial \vec{S}_{n}}{\partial t}=\gamma_{n} \cdot\left(\overrightarrow{B_{n}}+\vec{L}\right) \times \vec{S}_{n}+\vec{\Omega} \times \vec{S}_{n}+R_{s e}^{n e}\left(\vec{S}_{e}-\vec{S}_{n}\right)-R_{t o t}^{n} \vec{S}_{n} \\
& \frac{\partial \vec{S}_{e}}{\partial t}=\gamma_{e} \cdot\left(\overrightarrow{B_{e}}+\vec{L}\right) \times \vec{S}_{e}+\vec{\Omega} \times \vec{S}_{e}+\left(R_{p} s_{p}+R_{s e}^{e n} \vec{S}_{n}+R_{m} S_{m}-R_{t o t}^{e} \vec{S}_{e}\right)
\end{aligned}
$$

$$
\begin{aligned}
& S_{x}^{e} \approx \frac{S_{z}^{e} \gamma_{e} R_{t o t}^{e}}{\left(R_{t o t}^{e}\right)^{2}+\left(\gamma_{e} B_{z}^{e}\right)^{2}} \cdot\left[b_{y}^{n}+b_{y}^{e}+L_{y}+\frac{\Omega_{y}}{\gamma_{n}}+\frac{S_{m} R_{m}+\alpha R_{p}}{\gamma_{e} S_{z}^{e}}\right. \\
& \left.+B_{z}^{e}\left(\frac{b_{y}^{e}+B_{y}^{e}}{B_{c}}+L_{x} \frac{\gamma_{e}}{R_{t o t}^{e}}\right)+\frac{\gamma_{e}}{R_{t o t}^{e}}\left(\frac{B_{x}^{e} B_{z}^{e}\left(B_{z}^{e}+L_{z}\right)}{B_{c}}+L_{x} L_{z}\right)\right]
\end{aligned}
$$

Here $\vec{S}_{n}$ is the polarization of nuclear spins belonging to the noble gas, and it contains three orthogonal components, $S_{x}^{n}, S_{y}^{n}$, and $S_{z}^{n} ; \overrightarrow{S_{e}}$ is the spin-polarization of the outer most electrons belonging to alkali-atoms, and it contains three orthogonal components, $S_{x}^{e}, S_{y}^{e}$, and $S_{z}^{e} ; S_{x}^{e}$ is the spin-polarization of the outermost electrons belonging to alkali-atoms along the $\mathrm{x}$ direction, which can be used to describe the output signal of the equipment; $\gamma_{e}$ is the gyromagnetic ratio of the electron; $\gamma_{n}$ is the gyromagnetic ratio of the noble gas; $\vec{B}_{e}$ is the magnetic field detected by electrons belonging to alkali-atoms, and it contains three components, $B_{x}^{e}, B_{y}^{e}$, and $B_{z}^{e} ; \vec{B}_{n}$ is the magnetic field detected by nuclei belonging to the noble gas; $B c$ is the magnetic field produced by the alkali atoms and the noble gas; $\vec{\Omega}$ is the rotating angular velocity, which describes the relative motion between carrier coordinate system and inertial coordinate system, and it contains three orthogonal components, $\Omega_{x}, \Omega_{y}$, and $\Omega_{z} ; R_{s e}^{n e}$ is the spin-exchange rate between the electron spins of alkali-atoms and the nuclear spins of the noble gas; $R_{t o t}^{n}$ is the total relaxation of the nuclear spins belonging to the noble gas; $R_{t o t}^{e}$ is the total relaxation of the electron spins; $R_{P}$ is the average rate at which un-polarized atoms absorb photons from the pump beam; the circular polarization degree of pump light is defined as $S_{P}$, and $S_{P}=1 ; R_{m}$ is the average rate at which un-polarized atoms absorb photons from the probe beam; the circular polarization degree of probe light is defined as $S_{m}$, and $S_{m}=0 ; \vec{L}$ is defined as light shift, which is equivalent to the virtual magnetic field, and it contains three orthogonal components, $L_{x}, L_{y}$, and $L_{z} ; b$ is defined as an anomalous field; $\alpha$ is the non-orthogonal angle between pump beam and probe beam, and when the probe beam is perpendicular to the pump beam, the value of $\alpha$ is zero. Because several parameter values are very small, the output signal can be simplified as follow.

$$
S_{x}^{e} \approx \frac{S_{z}^{e} \gamma_{e} R_{t o t}^{e}}{\left(R_{t o t}^{e}\right)^{2}+\left(\gamma_{e} B_{z}^{e}\right)^{2}} \cdot \frac{\Omega_{y}}{\gamma_{n}}
$$

Eq. (4) shows that the ASIMD is based on the pattern of the angular rate model. Because the device is a new type of inertial measurement device, there are no mature standards for the scale factor calibration. According to the features of the ASIMD, a test scheme is designed to calibrate the scale factor for the device. The scale factor is always 
used in the inertial technology, as shown in the following equation.

$$
\Omega=K \cdot V
$$

Here $\mathrm{V}$ is the output voltage signal of the ASIMD, and its unit is $\mathrm{V} ; \Omega$ is the angular rate signal of the carrier detected by the ASIMD, and it is the input signal with the unit $\%$; $\mathrm{K}$ is the scale factor, and its unit is $\% / \mathrm{s} / \mathrm{V}$.

The original output signal of the device is a voltage signal, which needs to be converted to an angular rate signal to match with the input signal. The inertial measurement unit (IMU) based on interference fiber optic gyros (IFOGs) can be used to assist the atomic spin inertial measurement device to implement this function. The atomic spin inertial measurement device and the IMU are fixed on the same optical platform. The IMU consists of three IFOGs based on angular rate model and three accelerometers, which can be used to measure the carrier motion information including translational motion and rotary motion in three perpendicular directions.

\section{EXPERIMENTAL SETUP AND RESULTS}

\subsection{Experimental Setup}

The ASIMD (the size of the ASIMD is $1800 \mathrm{~mm}$ (Length) $\times 1500 \mathrm{~mm}$ (Width)) was installed on the optical platform. The optical platform can be supported through the six airbearing legs, which makes the device rotate around the support legs slightly. Because spaces available for motion of the gasbags of the legs are limited, the maximum rotation angle is about $\pm 2^{\circ}$. In order to provide a measurement criterion of angular velocity for the atomic spin inertial measurement device, high precision fiber optic IMU was fixed on the optical platform together with the atomic spin inertial measurement unit. High precision fiber optic gyroscopes can measure

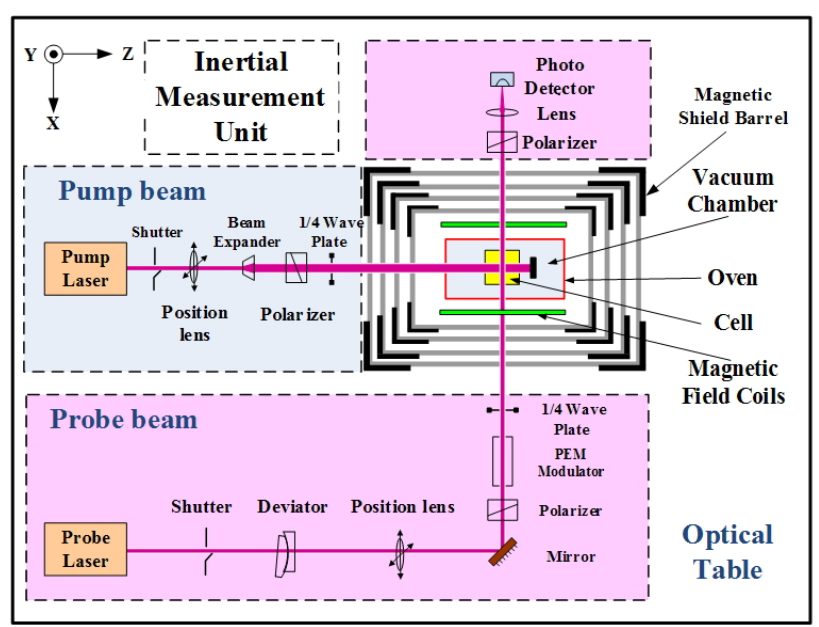

FIG. 1. Schematic diagram of experimental device.

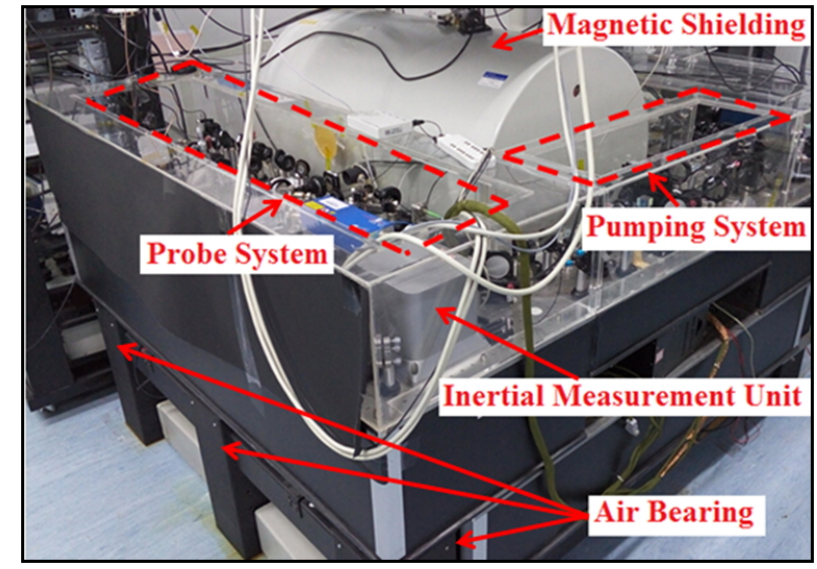

FIG. 2. Photograph of experimental device.

the angular velocity of the device, playing an important part in calibrating the output signal of the atomic spin inertial measurement device. The test hardware structure diagram and the physical graph are shown as follows.

\subsection{Results and Error Analysis}

The optical platform was driven around the y-axis with small amplitude. The results of angular rate along the y-axis detected by the high precision optical fiber gyroscopes (the sampling frequency of the gyroscope is $200 \mathrm{~Hz}$ and the bias instability of the gyroscope is $0.03 \%$ ) and the ASIMD were shown as follows. The output results of the two kinds of devices were subjected to least-squares model fitting, as shown in Fig. 3.

Making use of scale factor calibration coefficient, the output signal of ASIMD can be transformed into the angular velocity from the voltage signal, which is in contrast to IMU in Fig. 4. There are many factors causing the errors and they can be mainly divided into two categories.

Firstly, because the experimental platform is supported by the air-bearing legs, the driven amplitude of the optical platform is very small. The maximum driven amplitude of the optical platform was about $0.2^{\circ}$, as seen in Fig. 4. Under this condition, the random errors have a great influence on the results of the experiment, and it led to the inaccuracy of the scale factor.

Secondly, the driven frequency of the platform cannot be controlled accurately. According to the amplitude-frequency curve, most vibration frequencies of the optical platform were less than $5 \mathrm{~Hz}$, and the driven frequency was mainly focused on $1.133 \mathrm{~Hz}$, as shown in Fig. 5. The bandwidth of the ASIMD needs to be confirmed, which may influence the experimental results. According to the Fig. 6, the adjustment time of the system is about $0.4 \mathrm{~s}$. The bandwidth $(0 \mathrm{~dB})$ of the ASIMD is less than $2.5 \mathrm{~Hz}$. The signal amplitude whose frequency was higher than $2.5 \mathrm{~Hz}$ has been attenuated because of the bandwidth limit of the ASIMD. Under this condition, the scale factor calibration of the ASIMD may be inaccurate. 


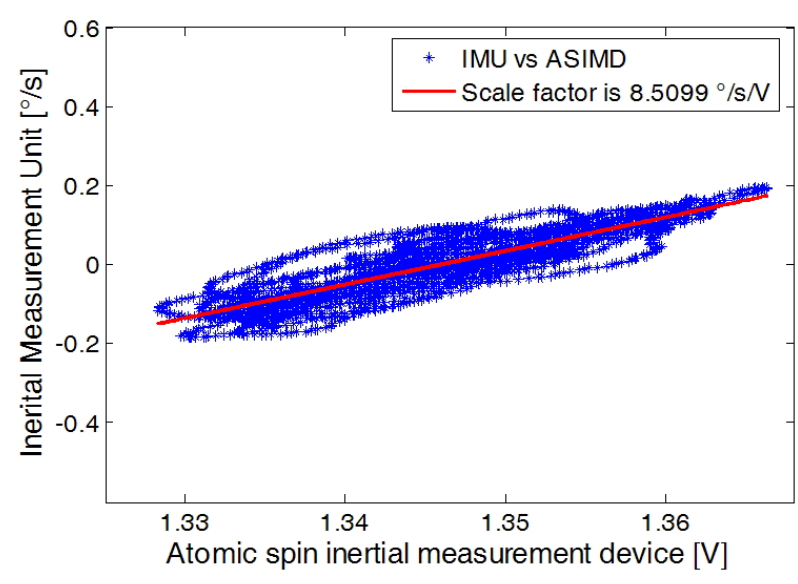

FIG. 3. Scale factor calibration of ASIMD based on the IMU.

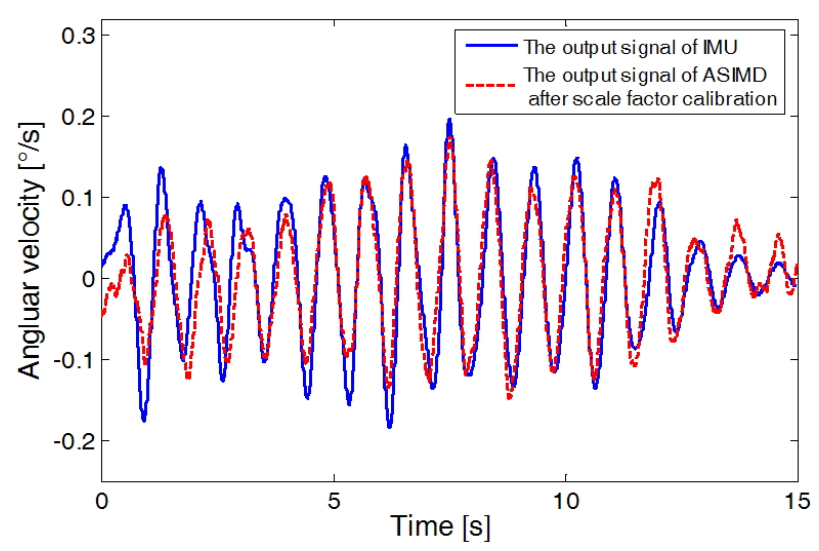

FIG. 4. Comparisons of output signal the between IMU and ASIMD after scale factor calibration.

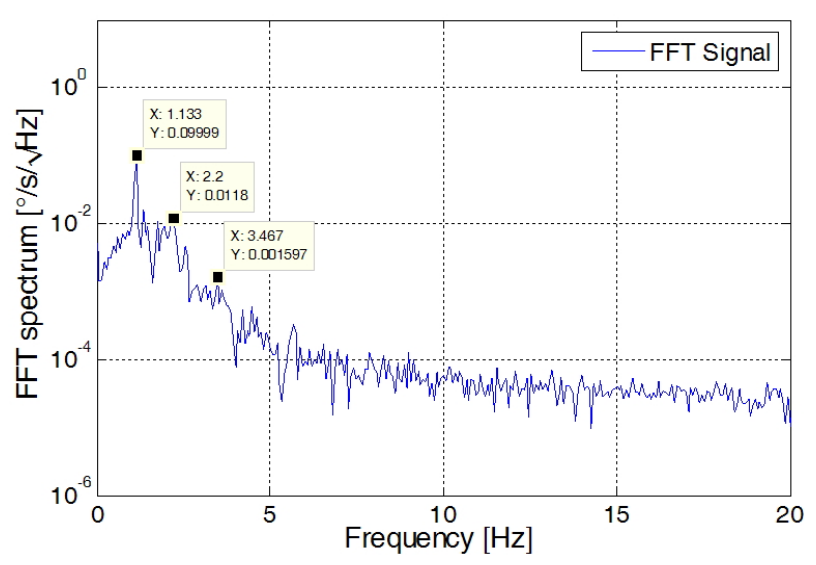

FIG. 5. Vibration frequency of the optical platform.

\subsection{Experimental Improvement}

According to the Eq. (1) to (3), the right hand side of the Eq. (3) can be simplified and merged. Thus, the output signal of the ASIMD can be simplified into the following expression.



FIG. 6. Response to the step signal from the ASIMD.

$$
\begin{aligned}
& S_{x}^{e} \approx \frac{S_{z}^{e} \gamma_{e} R_{t o t}^{e}}{R_{t o t}^{e}+\left(\gamma_{e} B_{z}^{e}\right)^{2}} \cdot \frac{1}{\gamma_{n}} \Omega_{y}+\frac{S_{z}^{e} \gamma_{e} R_{t o t}^{e}}{R_{t o t}^{e}+\left(\gamma_{e} B_{z}^{e}\right)^{2}} \cdot\left[L_{y}+\frac{S_{m} R_{m}+\alpha R_{p}}{\gamma_{e} S_{z}^{e}}+\right. \\
& \left.B_{z}^{e}\left(\frac{b_{y}^{e}+B_{y}^{e}}{B_{c}}+L_{x} \frac{\gamma_{e}}{R_{t o t}^{e}}\right)+\frac{\gamma_{e}}{R_{t o t}^{e}}\left(\frac{B_{x}^{e} B_{z}^{e}\left(B_{z}^{e}+L_{z}\right)}{B_{c}}+L_{x} L_{z}\right)\right]
\end{aligned}
$$

According to the Eq. (5), the scale factor can be described with the letter $K$. The magnetic shield barrels shield the external magnetic field and the magnetic coils further offset the residual magnetic field inside the barrels. So the residual magnetic field in three different directions $\left(B_{x}=B_{y}=B_{z} \approx\right.$ $0 L_{x}=L_{y}=L_{z} \approx 0$ ) approaches zero under the actual condition. According to the Eq. (4) to (5), the scale factor $K$ can be described with the Eq. (7).

$$
K=\frac{R_{t o t}^{e}}{S_{z}^{e} \gamma_{e}} \cdot \frac{\gamma_{n}}{1}
$$

According to the output signal of the ASIMD which is shown in Eq. (4), we took its partial respect to the magnetic field $B_{y}$, as shown in Eq. (8). The actual data of the left hand side in Eq. (8) can be obtained by the experiments.

$$
\frac{D S_{x}^{e}}{d B_{y}}=\frac{S_{z}^{e} \gamma_{e} R_{t o t}^{e}}{R_{t o t}^{e}+\left(\gamma_{e} B_{z}^{e}\right)^{2}} \cdot \frac{B_{z}^{e}}{B_{c}}
$$

Based on the above theories, we carried out related experimental research work. The core sensitive header of the device was a square glass cell with a side of length $2 \mathrm{~cm}$. The cell, containing a droplet of cesium, 5 torr xenon 129 and $1 \mathrm{~atm}$ nitrogen, was heated to $100{ }^{\circ} \mathrm{C}$ with average relative error of $0.5{ }^{\circ} \mathrm{C}$. The power of the pumping light was $100 \mathrm{~mW}$. The power of the probe light was $4 \mathrm{~mW}$. After about one hour, the system had reached steady working condition. In order to get the signal $D S_{x}^{e} / d B_{y}$, 


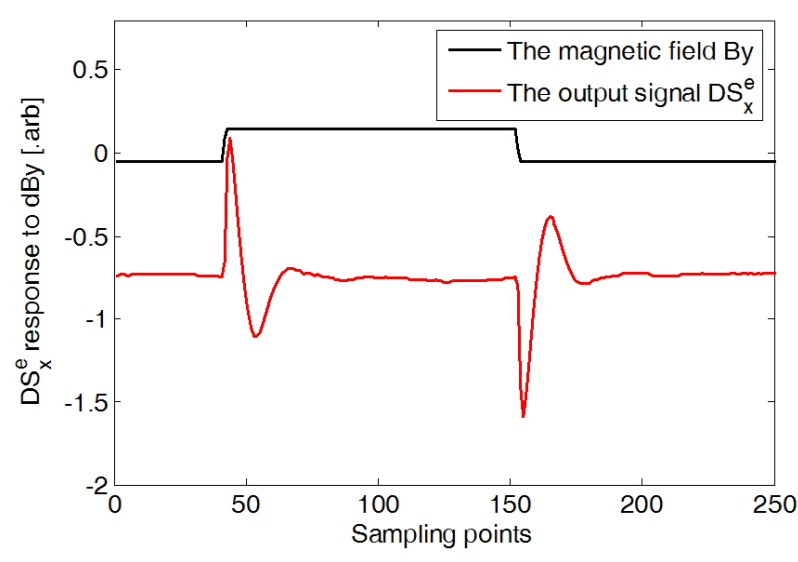

(a)



(b)

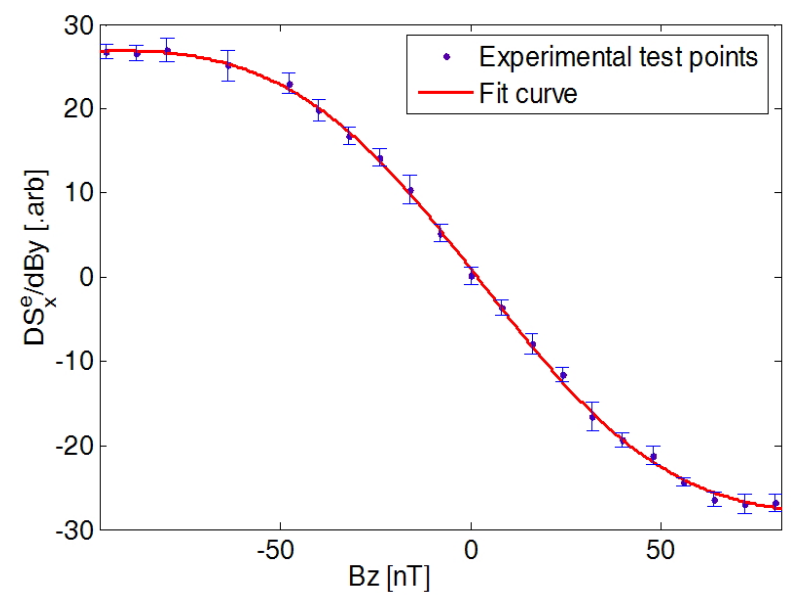

(c)

FIG. 7. $D S_{x}^{e}$ response to $d B_{y}$ under the situation of modulating $B_{z}$ step by step. (a) The situation $D S_{x}^{e} / d B_{y}=0$, (b) The situation $D S_{x}^{e} / d B_{y}>0$, (c) The experimental results of self-consistent scale factor calibration for ASIMD.

we modulate the magnetic field $B_{z}$ under the square wave magnetic field $B_{y}$ with the amplitude of $3 \mathrm{nT}$ and frequency of $90 \mathrm{mHz}$ to get the output signal. The experimental results of the situation $D S_{x}^{e} / d B_{y}>0$ are shown in Fig. 7(a). Because the situation $D S_{x}^{e} / d B_{y}<0$ is similar to the
TABLE 1. The experimental results for parameters fitting

\begin{tabular}{c|c|c|c|c}
\hline Fumber of trial & $\mathrm{a}$ & $\mathrm{b}$ & $\begin{array}{c}\text { Scale } \\
\text { factor } \\
(\mathrm{V} / \%)\end{array}$ & $\begin{array}{c}\mathrm{R}- \\
\text { square }\end{array}$ \\
\hline 1 & -5000 & -92.18 & 7.205 & 0.9989 \\
\hline 2 & -5018 & -92.38 & 7.210 & 0.999 \\
\hline 3 & -5044 & -92.7 & 7.223 & 0.9992 \\
\hline 4 & -5159 & -94.07 & 7.272 & 0.9994 \\
\hline 5 & -5159 & -94.07 & 7.272 & 0.9994 \\
\hline Errors (\%) & $1.61 \%$ & $1.05 \%$ & $0.49 \%$ & - \\
\hline
\end{tabular}

situation $D S_{x}^{e} / d B_{y}>0$, we only displayed the situation $D S_{x}^{e} / d B_{y}>0$. The experimental results of the situation $D S_{x}^{e} / d B_{y}=0$ are shown in Fig. 7(b). The whole experimental results of the scale factor calibration for the device are shown in Fig. 7(c). The scale factor can be got from the fit curve in Fig. 7(c).

According to the Eq. (7) to (8), the experimental test points can be used to fit a curved line. The scale factor can be determined by the fitting coefficients $a b$, and $c$, as shown in Eq. (9) to (10).

$$
\begin{aligned}
& y=\frac{a^{*}(x-x 0)}{(x-x 0)^{2}+b^{2}}+c \\
& K=\left|\frac{b^{2}}{a} \cdot \gamma_{n}\right|=\frac{R_{t o t}^{e} \gamma_{n}}{S_{z}^{e} \gamma_{e}}
\end{aligned}
$$

The fitting results of the experiments are shown in Table 1. Choosing coefficient of determination (R-square) as the judging standard, the best results of the two important fitting coefficients were $-5159,-94.07$ for $a$ and $b$, respectively. The gyromagnetic ratio belonging to xenon-129 is 4.2397 $\% / n T$, and the calibration result for scale factor was $7.272 \mathrm{~V} / \% / \mathrm{s}$ by multi-group experiments with the maximum error value $0.49 \%$.

\section{CONCLUSION}

In this paper, we calibrated the scale factor for the ASIMD device by using a new method, which utilized its self-signal rather than other calibration equipment to determine the scale factor with small errors. At first, the IMU was used to calibrate the scale factor for the ASIMD. However, the output signal of the ASIMD always fell behind the output signal of the IMU because of the limits of the ASIMD's bandwidth. Then, the method to determine the scale factor in a self-consistent way by using the gyromagnetic ratio of the noble gas was presented, and it has been verified by multi-group experiments. 
In the future, we intend to implement an inertial measurement with different spin-polarized noble gases and to find the most suitable noble gas for our application.

\section{ACKNOWLEDGMENT}

The authors would like to thank Profs. Jian-cheng Fang and Xi-yuan Chen for valuable suggestions and helpful discussions. This work is supported by the scientific research foundation of graduate school of Southeast University YBJJ1536 and the special fund for basic research on scientific instruments of the national science foundation of China under Grant No. 61227902.

\section{REFERENCES}

1. F. Allmendinger, W. Heil, S. Karpuk, W. Kilian, A. Scharth, U. Schmidt, A. Schnabel, Y. Sobolev, and K. Tullney, "New limit on lorentz-invariance- and CPT-violating neutron spin interactions using a free-spin-precession He-3-Xe-129 comagnetometer," Phys. Rev. Lett. 112, 110801 (2014).

2. S. J. Kim, J. J. Noh, M. S. Kim, J. S. Lee, H. Yu, and J. B. Kim, "Dynamics of a Bose-Einstein condensate on changing speeds of an atomchip trap potential," J. Opt. Soc. Korea 18, 633-638 (2014).

3. K. Kim, S. Begus, H. Xia, S. Lee, V. Jazbinsek, Z. Trontelj, and M. V. Romalis, "Multi-channel atomic magnetometer for magnetoencephalography: A configuration study," Neurolmage 89, 143-151 (2014).

4. B. Patton, E. Zhivun, D. C. Hovde, and D. Budker, "All-optical vector atomic magnetometer," Phys. Rev. Lett. 113, 13001 (2014).

5. J. Fang and J. Qin, "Advances in atomic gyroscopes: A view from inertial navigation applications," Sensors 12, 63316346 (2012).

6. D. Tazartes, "An historical perspective on inertial navigation systems," in Proc. The Inertial Sensors and Systems (ISISS), 2014 International Symposium on (Laguna Beach, CA, USA, Jan. 2014).

7. J. Kitching and S. Knappe, and E. A. Donley, "Atomic sensors - A review," IEEE Sensors Journal 11, 1749-1758 (2011).

8. P. D. Groves, "The PNT boom: Future trends in integrated navigation," Inside GNSS 8, 44-49 (2013).

9. J. C. Allred, R. N. Lyman, T. W. Kornack, and M. V. Romalis, "High-sensitivity atomic magnetometer unaffected by spin-exchange relaxation," Phys. Rev. Lett. 89, 130801 (2002).

10. D. Budker, W. Gawlik, D. Kimball, S. Rochester, V. Yashchuk, and A. Weis, "Resonant nonlinear magneto-optical effects in atoms," Reviews of Modern Physics 74, 1153-1201 (2002).

11. M. S. Larsen, H. C. Abbink, T. G. Walker, and M. D. Bulatowicz, "Nuclear magnetic resonance probe system," Google Patents (2013).

12. T. W. Kornack, R. K. Ghosh, and M. V. Romalis, "Nuclear spin gyroscope based on an atomic comagnetometer," Phys. Rev. Lett. 95, 23080123 (2005).

13. R. P. Moeller, W. K. Burns, and N. J. Frigo, "Open-loop output and scale factor stability in a fiber-optic gyroscope," IEEE J. Lightwave Technol. 30, $262-269$ (1989).

14. J. C. Fang, X. Zhang, and J. L. Li, "A compensation method for MEMS gyro scale factor error," Acta Aeronautica et Astronautica Sinica 02, 350-355 (2010), in Chinese.

15. C. Shen and X. Y. Chen, "Analysis and modeling for fiber-optic gyroscope scale factor based on environment temperature," Appl. Opt. 51, 2541 (2012).

16. H. Chung, L. Ojeda, and J. Borenstein, "Accurate mobile robot dead-reckoning with a precision-calibrated fiber-optic gyroscope," IEEE Transactions on Robotics and Automation 17, 80-84 (2001). 